\title{
Bénard-Marangoni convection in square containers
}

\author{
D. Krmpotić, ${ }^{1}$ G. B. Mindlin, ${ }^{2}$ and C. Pérez-García ${ }^{3}$ \\ ${ }^{1}$ Departamento de Física, Universidad Nacional de La Plata, La Plata, Argentina \\ ${ }^{2}$ Departamento de Física, Facultad de Ciencias Exactas y Naturales, Ciudad Universitaria, Pab. I, Código Postal 1428, Buenos Aires, \\ Argentina \\ ${ }^{3}$ Departamento de Física y Matemática Aplicada, Facultad de Ciencias, Universidad de Navarra, 31080 Pamplona, Navarra, Spain
}

(Received 5 October 1995; revised manuscript received 10 January 1996)

\begin{abstract}
Convection in a square container of small aspect ratio is studied taking into account thermocapillarity as well as gravity effects. In addition to the geometrical symmetry $\left(D_{4}\right)$, the existence of hidden translational symmetries, due to boundary conditions, allows us to explain the qualitative features of the patterns found in recently reported experiments [T. Ondarzuhu et al., Phys. Rev. Lett. 70, 3892 (1993)]. The nonlinear interaction between mixed modes and pure modes is shown to give rise to a sequence of bifurcations that leads to the onset of oscillations, as observed experimentally. [S1063-651X(96)09006-X]
\end{abstract}

PACS number(s): 47.27.Cn, 47.27.Nz

\section{INTRODUCTION}

Pattern formation is an area of active research in a wide variety of extended physical systems [1]. An important class of these systems appears after a convective instability [2]. In a fluid layer heated from below these systems organize themselves into convective cells, provided that the temperature difference between the bottom and the top of the container is beyond a critical value $\Delta T_{c}$. In pure liquids convection is due to buoyancy forces when the fluid is enclosed in a rigid container [Rayleigh-Bénard (RB) convection]. When the upper surface is open to the air surface tension provides a second instability mechanism [Bénard-Marangoni (BM) convection].

Convection leads to different patterns depending on the aspect ratio $a$, the ratio between a characteristic horizontal length and the liquid depth. For large values of $a$ the convective pattern is quite regular, although some defects can be present due to the side walls. For a small value of $a$ the lateral boundary conditions play an important role, which is not yet completely understood. In the case of RB convection in small aspect ratio containers some dynamical mechanisms (such as period doubling [3] and intermittency) have been observed experimentally. For BM convection some recent studies showed that the selection of modes is mainly due to lateral boundary conditions [4]. In a recent paper the pattern evolution in $\mathrm{BM}$ convection in a small aspect ratio cell $(a=4.46)$ has been reported [4]. In this experiment, a container with square insulating walls was filled with silicon oil of high Prandtl number. As the bottom temperature was increased from $35^{\circ} \mathrm{C}$ to $65^{\circ} \mathrm{C}$ the following sequence of bifurcations was observed. First, four convective cells appeared. When the temperature of the bottom plate was increased, the patterns broke symmetry and two square cells and two pentagonal ones were seen. Finally, with a further temperature increase the length of the segment joining the two square cells became time dependent.

The main purpose of this work is to obtain a set of equations that reflect the qualitative behavior seen in the experiment but starting from the Navier-Stokes equations as complementary to the phenomenological approach of the problem reported in [4]. In contrast with previous works on
BM convection we focus our attention in those modes that dynamically evolve to generate experimentally observed patterns. A more realistic approach to the problem was made by considering the relationship between two parameters $(M$ and $R$ ) that are physically related. Another important task of this work consists in studying the restrictions imposed by the geometry of vessels on the solution of the problem. In Sec. II the basic equations and the boundary conditions of this problem are discussed. Symmetry arguments allow us to select the modes compatible with these equations and geometrical constraints. Section III is devoted to the linear stability analysis focused on the modes that are permitted by symmetry and observed in experiments. The study is extended to the nonlinear regime in Sec. IV, and a comparison between the dynamical equations for the amplitude of these modes and the phenomenological model proposed [4] is made. Finally we comment on the disagreements between our idealized theoretical analysis and the experiment, and we discuss the main conclusions in Sec. V.

\section{EVOLUTION EQUATIONS AND BOUNDARY CONDITIONS}

The BM instability appears in a convective vessel heated from below with an upper free surface. Two different mechanisms contribute to the onset of instabilities: buoyancy and surface tension variations with temperature. The first mechanism is the usual one proposed by Lord Rayleigh to explain Bénard's observations. The second was proposed by Pearson [1]. Two facts makes BM convection more difficult to deal with theoretically than RB convection (no surface tension effects). First is the fact that the free interface has a dynamics driven by the stress and the second is that the heat exchange coefficient between the liquid and the air upon it is not well known [5]. Some articles prove that for sufficiently thick layers $(\sim 1 \mathrm{~mm})$ surface deflections can fall under the approximation (Oberbeck-Boussinesq approximation) taken in convective studies in liquids [6,7]. The second difficulty is usually surpassed by taking a phenomenological coefficient that accounts for those exchanges.

The basic state of the liquid is a purely conducting one where its temperature decreases linearly from the bottom to the upper surface. The nondimensional perturbations around the conducting state obey the following equations: 


$$
\begin{gathered}
\frac{M}{\operatorname{Pr}}\left\{\frac{\partial \mathbf{v}}{\partial t}+(\mathbf{v} \cdot \nabla) \mathbf{v}\right\}=\nabla^{2} \mathbf{v}-\nabla p+\frac{R}{M} \theta \hat{\mathbf{e}}_{3}, \\
\nabla \cdot \mathbf{v}=0, \\
M\left\{\frac{\partial \theta}{\partial t}-\omega+(\mathbf{v} \cdot \nabla) \theta\right\}=\nabla^{2} \theta,
\end{gathered}
$$

where $\mathbf{v}(x, y, z, t)=(u, v, \omega)$ is the velocity field and $\theta(x, y, z, t)$ the temperature perturbations. The parameters $R$, $M$, and $\operatorname{Pr}$ are nondimensional numbers, defined as $R=\alpha g d^{3} \Delta T / \kappa \nu$ (Rayleigh number), $M=\rho|d \sigma / d T| d \Delta T /$ $\nu \kappa$ (Marangoni number), and $\operatorname{Pr}=\nu / \kappa$ (Prandtl number). The two nondimensional numbers $R$ and $M$ are related by means of

$$
\gamma=M / R=\frac{\rho|d \sigma / d t|}{\alpha g d^{2}},
$$

which accounts for the relative importance of thermocapillary effects compared with gravity effects.

The system of Eqs. (2.1)-(2.3) is completed with the boundary conditions on the top (assumed undeformable and partially conducting) and the bottom (rigid and conducting) of the cell. These conditions can be written as

$$
\begin{gathered}
\mathbf{v}=\mathbf{0}, \quad \theta=0 \quad \text { on } z=0 \\
\omega=\frac{\partial u}{\partial z}+\frac{\partial \theta}{\partial x}=\frac{\partial \nu}{\partial z}+\frac{\partial \theta}{\partial y}=\frac{\partial \theta}{\partial z}+L \theta=0 \quad \text { on } z=1
\end{gathered}
$$

where the liquid-air interface is assumed to be flat and nonmoving. Here $L$ (Biot number) is a phenomenological parameter (for $L=0$ one has an insulating interface, while $L=\infty$ indicates a good heat conducting interface). In the following we will use in our calculations the values of $\gamma=0.01$ and $L=0.1$, which correspond to the experimental conditions of Ref. [8].

As we are considering a square and finite container, some lateral boundary conditions must be added. In the experiment considered in Sec. I, the lateral boundary conditions are rigid and insulating, but from the theoretical point of view it is convenient to introduce a simplifying assumption discussed by Rosenblat and co-workers [9]. These lateral boundary conditions are known as slippery conditions (impermeable, stress free, and adiabatic). These conditions are equivalent to assuming that (1) the lateral walls are impermeable, (2) the tangential vorticity is zero on them, and (3) they are adiabatic.

\section{Hidden symmetries}

The patterns observed experimentally are more symmetric than those predicted for a square vessel [10]. The $D_{4}$ symmetry alone does not explain why patterns that break the symmetry about $x \rightarrow a-x$ do preserve the symmetry about $y \rightarrow a-x$. The square symmetry is generated by two operations, namely, a reflection with respect to a diagonal $(x \leftrightarrow y)$, hereafter called $Z_{d}$, and a translation that changes $x \rightarrow a-x$, hereafter called $Z_{h}$. A transition between the first and the second patterns that appear in the experiment [4]

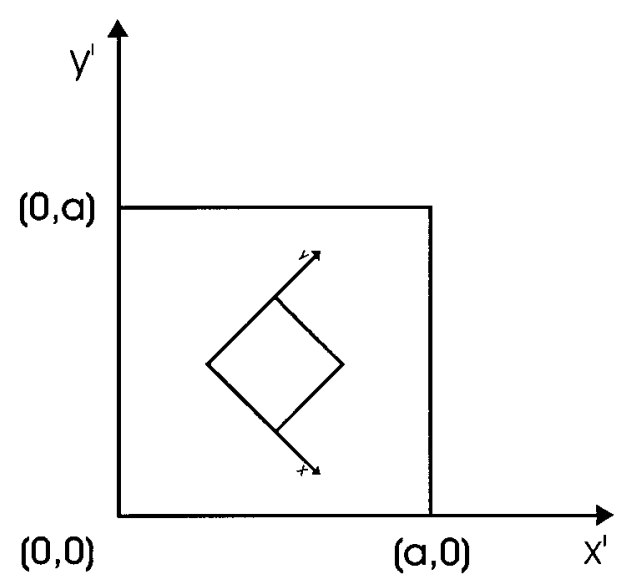

FIG. 1. Sketch of the transformation from a square container with Neumann boundary conditions to an enlarged and rotated square with periodic ones.

implies a breakup of the $Z_{h}$ symmetry, but not of the symmetry about $y \rightarrow a-x$. This symmetry remains because there is an additional hidden symmetry [11] due to the Neumann boundary conditions $(\hat{\mathbf{n}} \cdot \boldsymbol{\nabla} \theta=0)$ at $x=0, x=a, y=0$, or $y=a$ imposed by side walls. In fact, the existence of Neumann boundary conditions allows the solutions in the original domain $[(0,0) \leqslant(x, y) \leqslant(a, a)]$ to be extended to a greater one in which they satisfy periodic boundary conditions. This extended domain is a square with a side length twice the diagonal of the original, which is rotated an angle of $\pi / 4$ and translated in such a way that the centers of the original and extended squares coincide. The original problem can be thought of as the restriction of the problem with periodic boundary conditions in the extended square, to the small square. Now the solutions of the original problem have a translational symmetry, and reflection invariance about both diagonals becomes evident (see Fig. 1).

\section{LINEAR STABILITY ANALYSIS}

We proceed to examine the consequences of these symmetries on the form of the solutions of the linearized problem. Solutions of the linearized equations are taken in the form:

$$
\phi_{m n j}(x, y, z) \propto \cos (m \pi x / a) \cos (n \pi y / a) .
$$

Symmetries, however, impose the eigenfunctions to be invariant under the change $x \leftrightarrow y$ and, therefore, solutions should also be

$$
\begin{aligned}
\phi_{m n j}(x, y, z) \propto & \{\cos (m \pi x / a) \cos (n \pi y / a) \\
& +\cos (n \pi x / a) \cos (m \pi y / a)\} .
\end{aligned}
$$

(Notice that the latter includes the former as a particular case.) Modes with $m=n$ are also invariant under reflections with respect to the diagonal $y=a-x$. If this is not the case $Z_{d}$ and hidden symmetries impose that $m$ and $n$ must have the same parity for the permitted modes. We call pure modes those of the form (3.1) with $m=n$ and mixed modes those of the form (3.2) with $m \neq n$ and $m, n$ having the same parity.

The explicit forms of $\mathbf{v}$ and $\theta$ fields are 


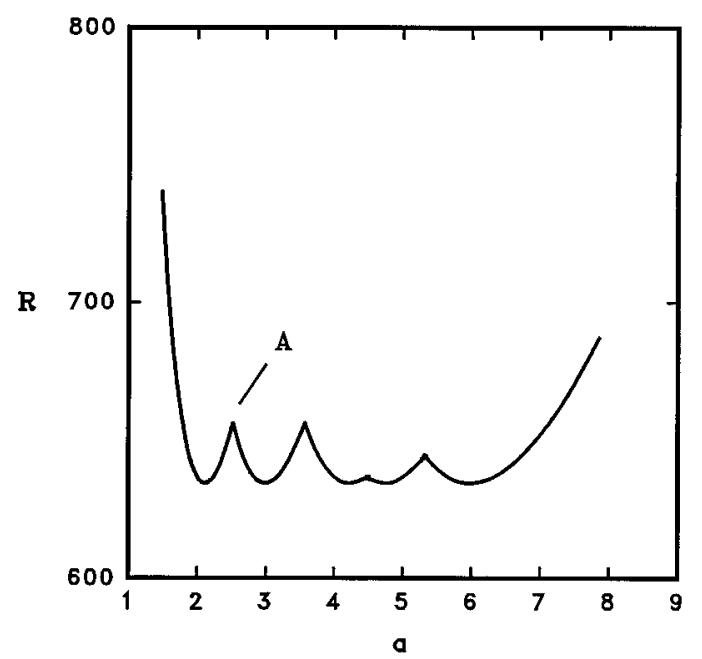

FIG. 2. Marginal equilibrium curves found for $\gamma=0.01$, $L=0.1$, for the modes $11,201,21,131$, and 401 . The point $A$ where two stationary mode interact is a codimension two point.

$$
\begin{aligned}
& \omega_{m m j}(x, y, z)=f_{m m j}(z) \cos (m \pi x / a) \cos (m \pi y / a), \\
& \theta_{m m j}(x, y, z)=g_{m m j}(z) \cos (m \pi x / a) \cos (m \pi y / a)
\end{aligned}
$$

for the pure modes and

$$
\begin{aligned}
\omega_{m n n m j}(x, y, z)= & f_{m n n m j}(z)\{\cos (m \pi x / a) \cos (n \pi y / a) \\
& +\cos (n \pi x / a) \cos (m \pi y / a)\} \\
\theta_{m n n m j}(x, y, z)= & g_{m n n m j}(z)\{\cos (m \pi x / a) \cos (n \pi y / a) \\
& +\cos (n \pi x / a) \cos (m \pi y / a)\}
\end{aligned}
$$

for the mixed modes, where $f_{\{i\}}(z)$ and $g_{\{i\}}(z)$ are the $z$ eigenfunctions of the linearized problem [9]. To simplify the notation we will retain for the first group only two indices $\{1\}=(m, j)$ and three for the second group $\{2\}=(m, n, j)$.

In the case studied here we chose as critical modes those whose linear superposition generates patterns similar to experimentally observed ones, namely,

$$
\theta_{201}(x, y, z)=g_{201}(z)[\cos (2 \pi x / a)+\cos (2 \pi y / a)]
$$

and

$$
\theta_{11}(x, y, z)=g_{11}(z) \cos (\pi x / a) \cos (\pi y / a)
$$

A linear combination of a mixed mode with $m=2, n=0, j=1$ and a pure one with $m=n=j=1$ resembles experimentally observed patterns [4].

The linear stability curves were found numerically using $R$ and $a$ as control parameters and finding the nontrivial $f_{\{i\}}(z)$ and $g_{\{i\}}(z)$ that also satisfy (2.5) excluding those that do not obey both $D_{4}$ and hidden symmetries (see Fig. 2).

\section{NONLINEAR EQUATIONS}

In order to obtain a finite-dimensional set of ordinary differential equations from the original partial differential equations we expand the fields $\theta$ and $\mathbf{v}$, using as a basis the critical and first subcritical eigenfunctions of the linearized problem.

The solution of the convective fields is assumed to be of the form [9]

$$
(\mathbf{v}, \theta)=\sum_{\{1,2\}} A_{\{1,2\}}(t)\left(\mathbf{v}_{\{1,2\}}, \theta_{\{1,2\}}\right)
$$

where $A_{\{1,2\}}(t)$ are the time-dependent amplitudes of the corresponding modes and $\mathbf{v}_{\{1,2\}}$ and $\theta_{\{1,2\}}$ are the fields in Eqs. (3.3)-(3.6)

From the solvability condition (Fredholm alternative) given by

$$
\begin{aligned}
\left(1-\frac{R_{c}}{R}\right)\left\langle\theta_{\{1,2\}}^{*} \omega^{\prime}\right\rangle+\frac{1}{\gamma^{2} R}\left(1-\frac{R_{\{1,2\}}^{*}}{r_{c}}\right)\left\langle\omega_{\{1,2\}}^{*} \theta^{\prime}\right\rangle \\
=\left\langle\theta_{\{1,2\}}^{*} \frac{\partial \theta^{\prime}}{\partial t}+\frac{1}{\operatorname{Pr}} \mathbf{v}_{\{1,2\}}^{*} \cdot \frac{\partial \mathbf{v}^{\prime}}{\partial t}\right\rangle+\left\langle\theta_{\{1,2\}}^{*}\left[\left(\mathbf{v}^{\prime} \cdot \nabla\right) \theta^{\prime}\right]_{t}\right\rangle \\
+\frac{1}{\operatorname{Pr}}\left\langle\mathbf{v}_{\{1,2\}}^{*} \cdot\left[\left(\mathbf{v}^{\prime} \cdot \nabla\right) \mathbf{v}^{\prime}\right]_{t}\right\rangle \\
+\gamma R\left\{\left\langle\theta_{\{1,2\}}^{*}\left(\overline{\omega^{\prime} \theta^{\prime}}\right)\right\rangle-\frac{1}{a^{2}} \frac{L}{L+1}\left\langle\theta_{\{1,2\}}^{*} \omega^{\prime}\right\rangle\left\langle\omega^{\prime} \theta^{\prime}\right\rangle\right\},
\end{aligned}
$$

we obtain the evolution equations for the chosen modes. In the last equation the brackets denote volume integral, the overbar denotes averaged quantities, the prime denotes departures from mean values, and an asterisk refers to solutions and parameters of the adjoint problem (see [13] for details). The development (4.1) will include the critical modes and the first subcritical ones near the codimension-two point A $\left(R=R_{c}, a=a_{c}\right)$ with $R_{c}=656.2$ and $a_{c}=2.526$ (see Fig. 2 ). The full ensemble of first damped modes and the corresponding eigenvalues are listed in Table I.

After performing a center manifold reduction [15] we arrive at two-dimensional set of ODE's for the amplitudes of 11 and 201 given by

$$
\begin{aligned}
& \dot{A}_{11}=\mu_{1} A_{11}+a_{1} A_{11} A_{201}+b_{1} A_{11}^{3}+c_{1} A_{11} A_{201}^{2}, \\
& \dot{A}_{201}=\mu_{2} A_{201}+a_{2} A_{11}^{2}+b_{2} A_{11}^{2} A_{201}+c_{2} A_{201}^{3},
\end{aligned}
$$

where the nonlinear terms in (4.3) are obtained from (4.2) assuming that $R=R_{c}$ and the numerical values of $a_{i}, b_{i}, c_{i}$ are listed in Table I(b). The coefficients $\mu_{1}$ and $\mu_{2}$ in (4.3) are related to physical control parameters by

$$
\begin{gathered}
\mu_{1}=\frac{\left(1-R_{c_{11}} / R\right)\left\langle\theta_{11}^{*} \omega_{11}\right\rangle}{\left\langle\theta_{11}^{*} \theta_{11}\right\rangle}, \\
\mu_{2}=\frac{\left(1-R_{c_{201}} / R\right)\left\langle\theta_{201}^{*} \omega_{201}\right\rangle}{\left\langle\theta_{201}^{*} \theta_{201}\right\rangle} .
\end{gathered}
$$

The unfolding of Eqs. (4.3) is made in a region slightly shifted to the right of point $A$ (Fig. 2). This is because ex- 
TABLE I. (a) Eigenvalues at the point $A$ in Fig. 2 for different modes, (b) coefficients of the nonlinear terms of the modes selected to make the center manifold reduction.

\begin{tabular}{lc} 
(a) & \\
$R_{11}^{*}$ & 656.2 \\
$R_{201}^{*}$ & \\
$R_{121}^{*}$ & 700.2 \\
$R_{131}^{*}$ & 1066.4 \\
$R_{231}^{*}$ & 1361.6 \\
$R_{101}^{*}$ & 851.2 \\
$R_{21}^{*}$ & 898.2 \\
$R_{401}^{*}$ & 1703.9 \\
& \\
(b) & \\
$a_{1}$ & \\
$b_{1}$ & 0.834 \\
$c_{1}$ & -1.793 \\
$a_{2}$ & -11.926 \\
$b_{2}$ & -0.144 \\
$c_{2}$ & -3.350 \\
\hline
\end{tabular}

perimentally the mode 201 first becomes unstable and from Fig. 2 one can easily see that for $a>a_{c}, R_{c_{201}}<R_{c_{11}}$. The sequence of bifurcations is thus obtained by moving along a line with $a_{\epsilon}=a+\epsilon(\epsilon>0)$. To the right of $A, \mu_{1}$ and $\mu_{2}$ will be constrained by the condition $\mu_{2}=\mu_{1}+\delta$ with $\delta>0$. Now we study the dynamics generated by Eqs. (4.3) when $R$ increases for a fixed $a=a_{\epsilon}$ near the codimension-two point. For $\mu_{1}$ and $\mu_{2}$ negative the only stable solution is the origin, i.e., the quiescent state. This is replaced by a state with $A_{201}$ when $R$ is slightly greater than $R_{c_{201}}$, and the second mode $A_{11}$ is subcritical. This state corresponds to a crosslike pattern $\left(A_{201} \neq 0, a_{11}=0\right)$ and it is stable between $0<\mu_{2}<\mu_{2 p}=7.82 \times 10^{-3}$. This is replaced by a mixed pattern $\left(A_{201}>A_{11} \neq 0\right)$ via a pitchfork bifurcation in the range $\mu_{2 p}<\mu_{2}<\mu_{2 h}=1.28 \times 10^{-2}$. Two fixed points, one with $A_{11}>0$ and the second $A_{11}<0$, become stable. Patterns in this situation break the $Z_{h}$ but conserve the $Z_{d}$ by construction. These points undergo a Hopf bifurcation at $\mu_{2}=\mu_{2 h}$ where the fixed points transfer their stability to limit cycles (see Fig. 3). In this case the diagonal of the pattern oscillates between a minimal and a maximal value. These values increase with $R$, but saturate, because the limit cycle cannot cross the $\left(A_{201}\right)$ axis.

\section{CONCLUSIONS}

We found a simple dynamical system starting from the hydrodynamic equations governing the motion of the fluid that qualitatively predicts experimentally observed phenomena. The Neumann boundary conditions for the temperature perturbations and the $z$ component of the velocity field explain the additional hidden symmetries present in this problem. A remarkable fact is that the sequence of modes that interact is only fixed by the geometry of the container and by
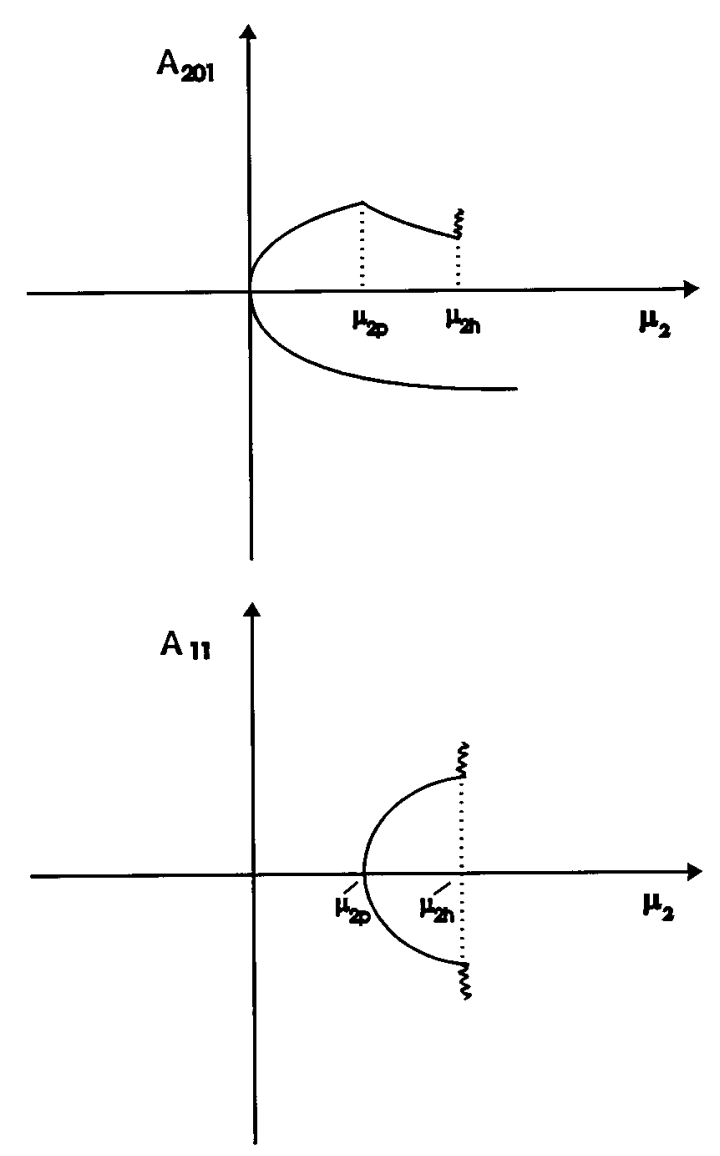

FIG. 3. Bifurcation diagrams for the amplitudes $A_{201}$ and $A_{11}$.

the thermal properties of the side walls (lateral boundary conditions) and not by the characteristics of the fluid or by the relative importance of Rayleigh and Marangoni convective mechanisms. This was also a conclusion found by other authors for pure thermocapillary convection [9].

The results on the amplitude dynamics are in qualitative agreement with experimental observations. The calculations reproduce the sequence of dynamical states and the platforms observed in the experiment, when the supercritical heating is increased.

However, an important quantitative difference subsists between our calculations and the experiment. The competition of two modes has been analyzed for the smallest aspect ratio $a$ where a crosslike pattern competes with a diagonallike pattern. In our calculations this occurs for $a=2.526$ while experiments have been performed for $a=4.46$. This discrepancy could be due to the important differences between the idealized boundary conditions used in our calculations and the real ones.

\section{ACKNOWLEDGMENTS}

This work has been partially funded by Fundación Antorchas (Argentina) and by the DGICYT (Spanish Government) under Contract No. PB93-0708, and by an UE Contract No. CI1*CT93-0331. G. B. Mindlin and D. Krmpotic would like to thank CONICET (Argentina) and CIC (Argentina) for financial support. 
[1] M. C. Cross and P. C. Hohenberg, Rev. Mod. Phys. 65, 851 (1993).

[2] E. L. Koschmieder, Bénard Cells and Taylor Vortices (Cambridge University Press, Cambridge, 1993).

[3] A. Libchaber and J. Maurer, J. Phys. (Paris), Colloq. 41, C3-51 (1980).

[4] T. Ondarçuhu, G. B. Mindlin, H. L. Mancini, and C. PérezGarcía, Phys. Rev. Lett. 70, 3892 (1993).

[5] A. Thess and S. Orszag, J. Fluid Mech. 283, 201 (1995).

[6] S. H. Davis and G. M. Homsy, J. Fluid Mech. 98, 527 (1980).

[7] C. Perez-Garcia and G. Carneiro, Phys. Fluids 3, 292 (1991).

[8] H. Mancini, Doctoral thesis, Universidad de Navarra (1994).

[9] S. Rosenblat, S. H. Davis, and G. M. Homsy, J. Fluid Mech. 120, 91 (1982); S. Rosenblat, G. M. Homsy, and S. H. Davis, ibid. 120, 123 (1982).

[10] M. Golubitsky, I. Stewart, and D. G. Schaeffer, Singularities and Groups in Bifurcation Theory II, Applied Mathematical Sciences Vol. 69 (Springer, New York, 1988).

[11] J. D. Crawford, Physica 52D, 429 (1991); J. D. Crawford, J. P. Gollub, and D. Lane, Nonlinearity 6, 119 (1993).

[12] D. A. Nield, J. Fluid Mech. 19, 341 (1964).

[13] P. C. Dauby, G. Lebon, P. Colinet, and J. C. Legros, Q. J. Mech. Appl. Math. 46, 683 (1983).

[14] S. Rosenblat, S. H. Davis, and G. H. Homsy, Phys. Fluids 24, 2215 (1981).

[15] J. Guckenheimer and P. Holmes, Nonlinear Oscillations, Dynamical Systems and Bifurcations of Vector Fields, Applied Mathematical Sciences, Vol. 42 (Springer, New York, 1983). 